\title{
A 2-DoF Skin Stretch Display on Palm: Effect of Stimulation Shape, Speed and Intensity
}

\author{
Ahmad Manasrah ${ }^{(\bowtie)}(1)$ and Shahnaz Alkhalil@ \\ Al Zaytoonah University of Jordan, Amman 11947, Jordan \\ \{ahmad.mansrah, shahnaz.k\}@zuj.edu.jo \\ https://sites.google.com/view/ahmadmanasrah/home
}

\begin{abstract}
Skin stretch has been widely utilized as a tactile display in different haptic applications. However, there has been little research focusing on skin stretch as a modality on the palm of the hand. In this study, a two dimensional tactor apparatus was designed and built to investigate the effects of stimulation speeds, shapes and intensities of skin stretch display on the palm. The tactor moved across the palm at different speeds to create stimulation shapes on the skin. Subjects reported the intensity of perceived stimuli and predicted speed rate of the tactor and stimulation shape and size. The results showed that there were statistically significant differences in the intensity of perceived tactile displays between different stimulation shapes and sizes. The results also showed the sizes and intensity of the stimulus grow larger with slower tactor speeds.
\end{abstract}

Keywords: Skin stretch $\cdot$ Palm $\cdot$ Stimulation intensity $\cdot$ Tactile display

\section{Introduction}

There have been significant advances in the field of tactile displays. The literature shows that tactile displays have the potential to improve the user's experience in gaming, teleoperation, and virtual environment simulation. For instance, tactile displays were utilized in delivering shape and material information to users [10] and, also, to deliver instructions and navigational commands [16]. Most of these haptic feedback technologies transfer information via vibrotactile actuators due to their effective perception on the skin and simple implementation $[3,7]$. However, the vibrotactile feedback does not necessarily provide directional information unless multiple actuators are utilized [17]. This type of tactile display may also create desensitization and discomfort to users at relatively high intensities $[21,26]$.

Recently, skin stretch has been introduced and implemented as an alternative tactile feedback modality in multiple studies. The skin stretch approach has the 


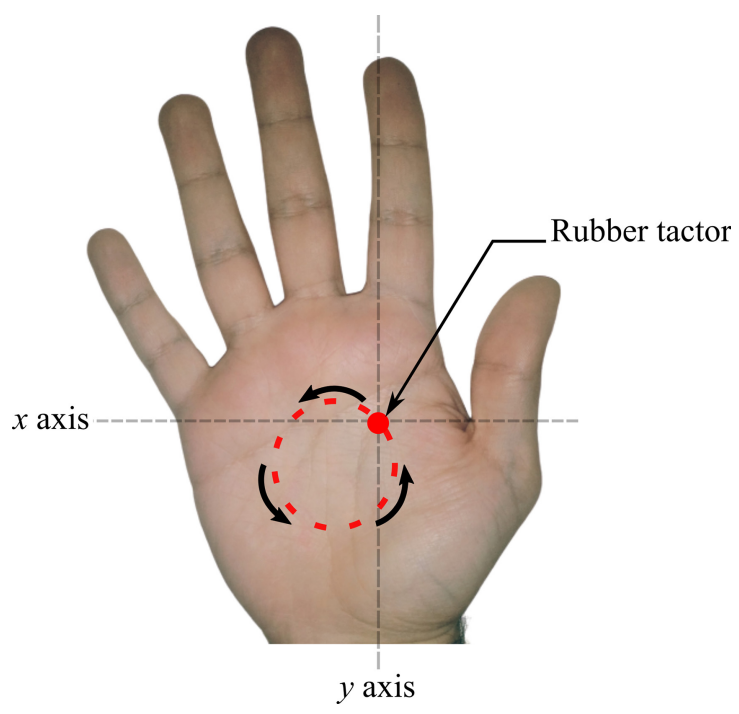

Fig. 1. Rubber tactor moves in two dimensions on the palm of the hand with different speeds to create a shape. In this case, a circle.

potential to deliver directional information especially with continuous stimuli using only one actuator [2]. The moving actuator applies a directional shear force on the surface of the skin, thus, activating the mechanoreceptors that are responsible of detecting and monitoring moving objects on the skin [15]. Shear forces, applied tangentially to the surface of the skin, result from the friction between the tip of the tactor and the skin [34]. Larger friction forces, for instance, trigger the mechanoreceptors with higher rates, thus increase the intensity of stimulation. In this study, we hypothesize that the speed of the actuator as well as the shape that is created on the palm of the hand can affect the user's perception of the intensity and area of stimulation. A two dimensional plotter mechanism was designed to move a rubber tactor across the skin as illustrated in Fig. 1. The haptic cue is conveyed when the tactor creates deformations of the skin in the direction at which it is traveling, hence, users can identify the drawn shapes and estimate their areas comparatively.

The purpose of this research is to investigate this hypothesis related to how users perceive tactile displays of different shapes and speeds. The results of this investigation may provide a new perspective on skin stretch as a method of delivering information and help the development of tactile display systems in many applications.

\section{Background}

Different tactile displays are perceived by different mechanoreceptors in the skin. Each type of mechanoreceptor is excited with the presence of certain stimuli. For 
instance, fast adapting receptors FA-II perceive the vibration stimulus with different sensitivities based on the frequency and the duration of the vibration [30]. Skin stretch stimuli, however, are perceived by the slowly adapting receptors SA-II [6]. These receptors stay active as long as there is a stimulus on the skin. Moreover, it has been shown that tangential forces caused by skin stretch can be perceived accurately and quickly by humans [4].

Skin stretch stimuli have been widely implemented in tactile display systems on different locations of the skin. Earlier studies have focused on the fingertip to investigate skin stretch as a modality to deliver information. For instance, a previous research showed that humans can differentiate between different tangential and normal forces when the stimulus is applied on the fingertip [20]. Gleeson et al, studied the effects of speed, displacement, and repetition of tangential skin stretch stimuli on the fingertip [12]. Their results showed that higher stimulus speeds improved the accuracy and perception of direction. Other studies investigated creating complex displays of virtual objects on the fingertip using skin deformation $[14,25]$. Yem et al. even produced a rubbing sensation on the fingertip using one degree of freedom movement [32].

There are also other studies that investigated skin stretch displays on the forearm [8] and on the lower extremities [7]. However, in comparison to other locations on the skin, few studies are found regarding skin deformation on the palm of the hand. Even though the palm is less sensitive than the fingertips, it has similar densities of SA-II receptors [29] and provides a larger display area for skin stretch stimulation [13]. Studies have shown that tactile displays which are applied on the fingers and the palm simultaneously improve users' perception of relatively large virtual objects [27]. Skin stretch stimuli on the palm have been used to deliver driving information and direction to drivers through the steering wheel [23].

Other studies have investigated several factors that may affect skin stretch and skin deformation. For example, Edin et al. applied tangential forces at different locations on the body including the palm of the hand. Their results proved that the speed and direction of the stimulus affect the perception of skin stretch displays [9]. A more recent study also showed that the intensity of skin stretch is affected by the speed and displacement of the stimulus [13]. The method presented here investigated how the intensity and size created by the stimulus were perceived based on different speeds and stimulation shapes.

\section{Method}

This investigation consisted of one set of experiments studying several tactor speeds and stimulation shapes. In each experiment, subjects perceived a random stimulation shape with a certain size and speed. Three stimulation shapes were used: a circle, a square, and an equilateral triangle at two different sizes and two tactor speeds. 


\subsection{Apparatus}

A two dimensional plotter was designed and built specifically for the purpose of this study. Unlike conventional 2D plotters, the stylus was replaced with a tactor and directed upwards so that the palm can rest on it. The tactor's tip was made out of rubber and had a spherical shape with a diameter of $4 \mathrm{~mm}$ which is about the size of a board pin. The plotter moved the tactor using two stepper motors attached in an H-bot connection capable of producing different speeds as shown in Fig. 2. The tactor was mounted on a 3D printer base that was connected to the plotter contact surface. All the internal components and connections of the two dimensional plotter were covered with a white box which was cut out in the center to allow the tactor to move freely in a $7 \times 7 \mathrm{~cm}$ space which is slightly less than the size of the average palm for an adult [24].

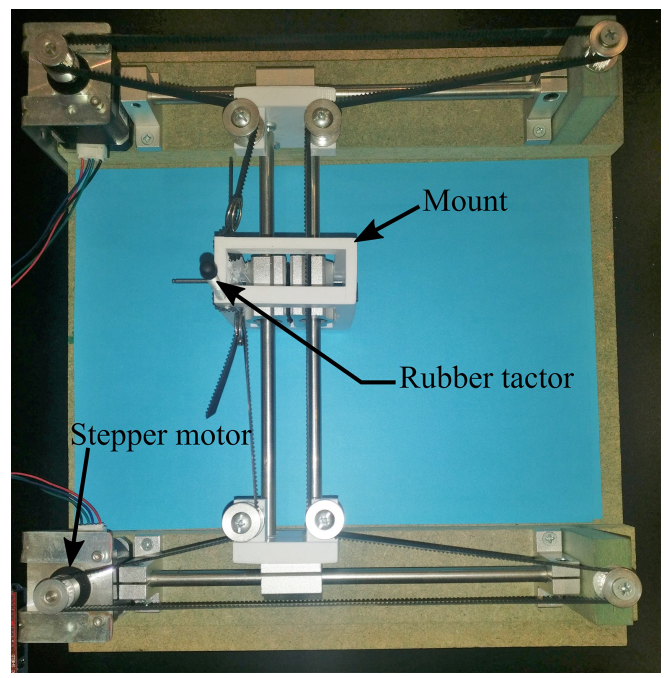

Fig. 2. Two dimensional plotter was built using two stepper motors. The rubber tactor is installed on the mount.

\subsection{Experimental Setup and Procedure}

At the beginning of the experiments, the nature of the study and procedures were generally explained to the subjects. After that, they were asked to sit in front of a screen and rest their right hand on the apparatus without applying extra force. Participants were also asked to wear headphones playing steady music to block noises generated from the apparatus and surroundings. Before the experiments began, a test-experiment was given to subjects where a stimulation shape is randomly applied on the participants' palms at two speeds $10 \mathrm{~mm} / \mathrm{s}$ and 20 $\mathrm{mm} / \mathrm{s}$. Participants were then asked to rate the intensity of stimuli on a scale 
from zero to four with zero being the weakest and four the strongest. The reason of conducting the test-experiment is to ensure that subjects had a reference intensity point to compare it to the stimuli that they were about to experience at the experiments.

During the experiments, three stimulation shapes were tested, a circle, square, and an equilateral triangle. Each shape was tested with two speeds $(10 \mathrm{~mm} / \mathrm{s}$ and $20 \mathrm{~mm} / \mathrm{s})$ and two areas. The stimulation areas were divided into "small" and "large" areas based on the shape. However since three stimulation shapes were used, the areas were not exactly equal for all the shapes. For instance, the "small" area of the circular stimulus was about $4.5 \mathrm{~cm}^{2}$ $(r=1.2 \mathrm{~cm})$, while as for the square, the area was $4.4 \mathrm{~cm}^{2}(w=2.1 \mathrm{~cm})$ and for the equilateral triangle the area was $1.7 \mathrm{~cm}^{2}(l=2 \mathrm{~cm})$. Table 1 shows the areas and perimeters for all three shapes.

Table 1. Stimulation shapes, speeds, and sizes.

\begin{tabular}{|c|c|c|c|}
\hline \multirow{2}{*}{ Shape } & \multirow{2}{*}{ Speed } & \multicolumn{2}{|c|}{ Size } \\
\hline & & Small & Large \\
\hline \multirow{2}{*}{ Circle } & $10 \mathrm{~mm} / \mathrm{s}$ & \multirow{2}{*}{$\begin{array}{c}r=1.2 \mathrm{~cm} \\
\text { Area }=4.5 \mathrm{~cm}^{2} \\
\text { Circumference }=7.5 \mathrm{~cm}\end{array}$} & \multirow{2}{*}{$\begin{array}{c}r=2.5 \mathrm{~cm} \\
\text { Area }=19.6 \mathrm{~cm}^{2} \\
\text { Circumference }=15.7 \mathrm{~cm}\end{array}$} \\
\hline & $20 \mathrm{~mm} / \mathrm{s}$ & & \\
\hline \multirow{2}{*}{ Square } & $10 \mathrm{~mm} / \mathrm{s}$ & \multirow{2}{*}{$\begin{array}{c}w=2.1 \mathrm{~cm} \\
\text { Area }=4.4 \mathrm{~cm}^{2} \\
\text { Perimeter }=8.4 \mathrm{~cm}\end{array}$} & \multirow{2}{*}{$\begin{array}{c}w=3.2 \mathrm{~cm} \\
\text { Area }=10.2 \mathrm{~cm}^{2} \\
\text { Perimeter }=12.8 \mathrm{~cm}\end{array}$} \\
\hline & $20 \mathrm{~mm} / \mathrm{s}$ & & \\
\hline \multirow{2}{*}{ Triangle } & $10 \mathrm{~mm} / \mathrm{s}$ & \multirow{2}{*}{$\begin{array}{c}l=2 \mathrm{~cm} \\
\text { Area }=1.7 \mathrm{~cm}^{2} \\
\text { Perimeter }=6 \mathrm{~cm}\end{array}$} & \multirow{2}{*}{$\begin{array}{c}l=3.5 \mathrm{~cm} \\
\text { Area }=5.3 \mathrm{~cm}^{2} \\
\text { Perimeter }=10.5 \mathrm{~cm}\end{array}$} \\
\hline & $20 \mathrm{~mm} / \mathrm{s}$ & & \\
\hline
\end{tabular}

With each experiment, a random stimulation shape was applied counterclockwise on the palm with a certain speed and size. The screen in front of the participants displayed all six stimulation shapes (two sizes for each shape). The sizes were exaggerated so that subjects can identify large and small shapes on the screen easily. The display also showed the intensity scale that was described previously. After each stimulation shape was applied, participants recorded their response using the information on the screen. A total of 12 shape, speed, and size combinations were randomly applied on each participant. The experiments took $15 \mathrm{~min}$ in total, including a few minutes break. Figure 3 shows the full experimental setup with the screen. 


\subsection{Participants}

A total of ten subjects participated in the experiments, six males and four females. All of them were healthy, right handed, and between the age of 18 and 50. Each subject read and signed a consent form agreeing to participate in the experiments.

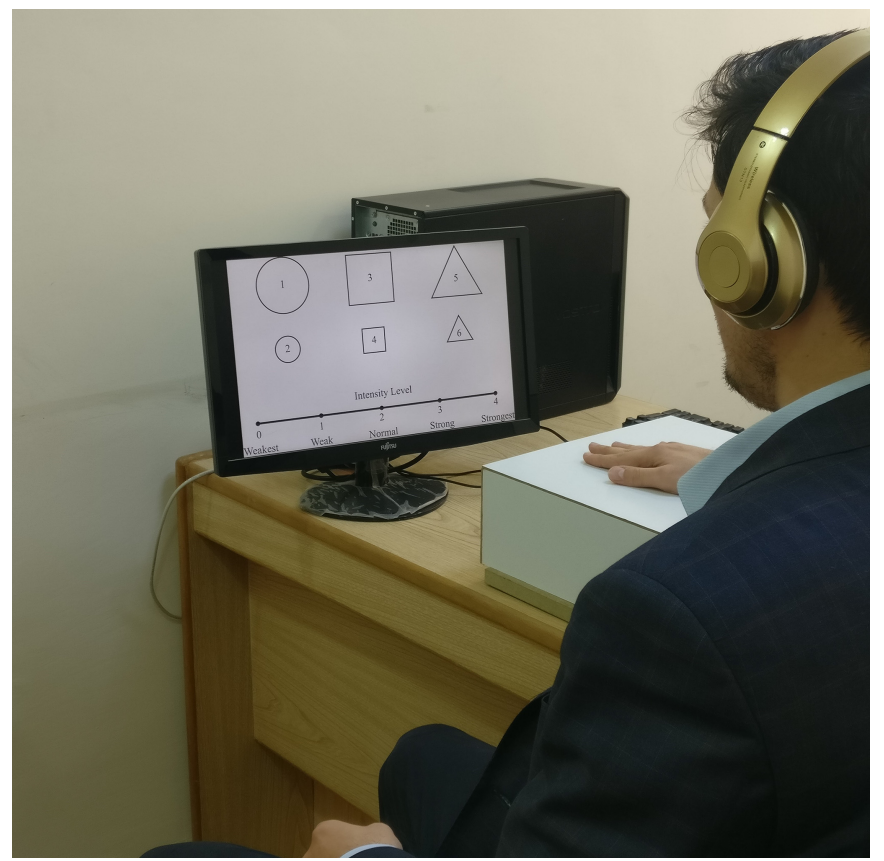

Fig. 3. Experiment setup where subject's right hand rests on the apparatus. The screen shows the stretch intensity scale and the shapes and sizes used in the experiments.

\section{Results}

In this study the collected data was analyzed using ANOVA with a dependent variable of stretch intensity and four independent variables of tactor speed, stimulation shape and size, and subject. Another ANOVA with a dependent variable of subject answers (shape, size) and three independent variables of stretch intensity, tactor speed, and subject was also conducted. When the results showed statistical differences, a Tukey's honest significant difference (HSD) test was performed as a post-hoc test. All statistical tests were based on alpha value of 0.05 .

The results of the first analysis showed that the "slow" tactor speed (10 $\mathrm{mm} / \mathrm{s})$ had a statistically significantly smaller stretch intensity $(F(1,106)=$ $58.06, p<0.001)$ than the "fast" stimuli $(20 \mathrm{~mm} / \mathrm{s})$ in all stimulation shapes. 


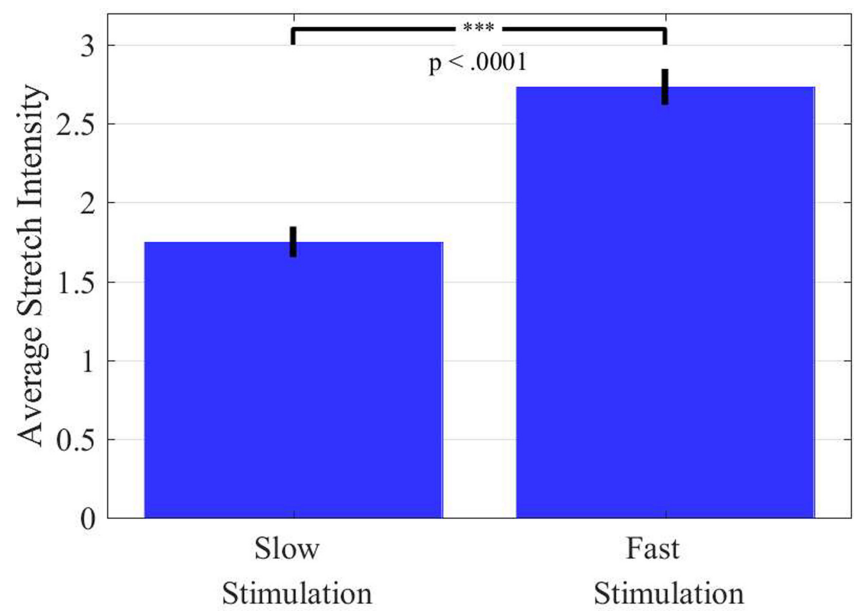

Fig. 4. The means and standard errors for the perceived stretch intensity at two tactor speeds. The average stretch intensity of slow tactor speeds was statistically significantly less than fast speeds.

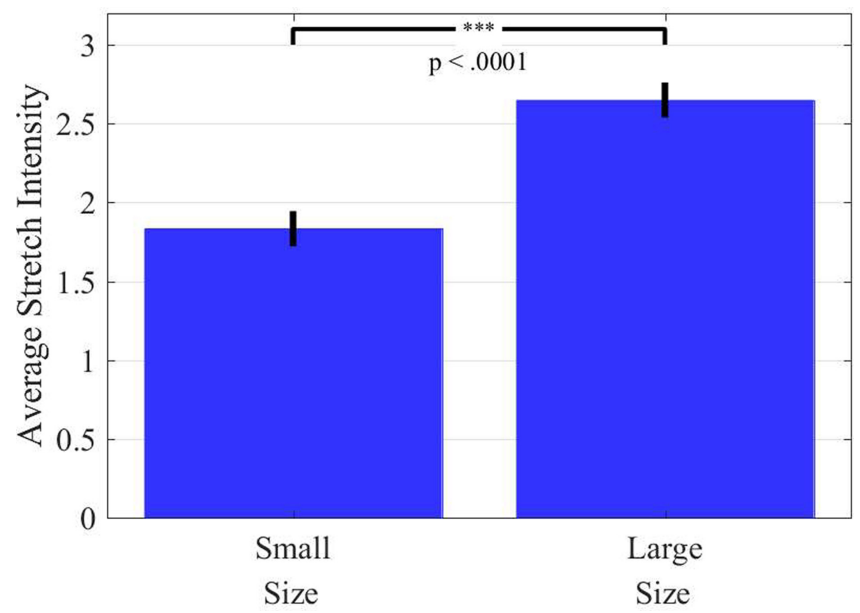

Fig. 5. The means and standard errors for the perceived stretch intensity at two stimulation sizes. The average stretch intensity of small stimulation sizes was statistically significantly less than large sizes.

Figure 4 shows the mean intensity of stimuli for the "slow" and "fast" tactor speeds. The slow tactor speed recorded a mean stretch intensity of 1.75 which was between "weak" and "normal". The mean stretch intensity of the fast tactor speed, however, was around 2.7, closer to "strong" on the stretch intensity scale. Moreover, the results showed that "small" stimuli sizes also had statistically significantly less stretch intensity $(F(1,106)=58.67, p<0.001)$ than "large" 
stimuli sizes within all the applied shapes as illustrated in Fig. 5. The results of stimuli shapes versus stretch intensity showed that the circular shape had statistically significantly less stretch intensity $(F(2,106)=26.27, p<0.001)$ than the other rectangular and triangular shapes regardless of the size. Figure 6 shows the stretch intensities of the three stimulation shapes. The results did not, however, show any statistical significant differences between subjects.

The second analysis focused on the perception of shapes and sizes of the stimuli. The results showed that subjects perceived the "slow" tactor speed statistically significantly "smaller" $(F(1,106)=15.79, p<0.0001)$ than the "fast" tactor speed as shown in Fig. 7. Furthermore, seven out of ten subjects missidentified the "small" square at "fast" tactor speed as a circle of the same size.

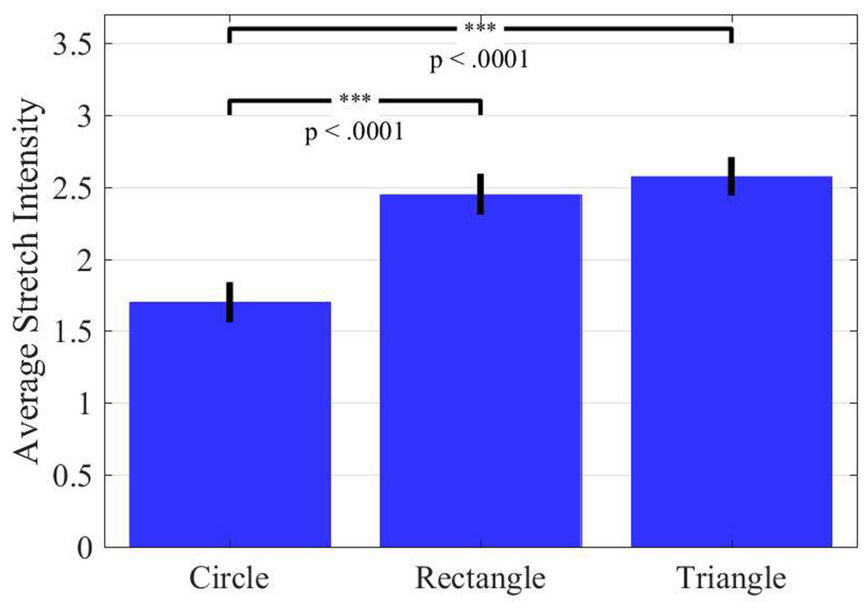

Fig. 6. The means and standard errors for the perceived stretch intensity at three stimulation shapes. The average stretch intensity of the circular shape was statistically significantly less than other stimulation shapes.

\section{Discussion}

In this study, the shape, speed, and intensity of skin stretch stimulation were investigated on the palm. The experimental results showed that the "slow" tactor speed was perceived with statistically significantly less intensity than the "fast" speed. These results agree with previous findings where the intensity of skin stretch perception increases as tactor speed increases [13]. They concluded that the relation between speed and intensity, however, was nonlinear. Other studies showed that an increase in the tactor speed enhances the intensity and accuracy of the perceived stimulus on the finger [12]. 
Further, the results of our experiments showed that "small" stimuli shapes were perceived with statistically significantly less intensity than "large" stimuli shapes. A small stimulation shape is created via short stimuli distance, thus, producing less stimulation intensity. Caswell et al. showed that shorter tactor displacement yielded a higher accuracy in predicting the direction of stimuli since their intensity increased [5]. The same results were also found in [13]. However, these findings can be related to the spatial distribution of the receptors perceiving the stimuli. Studies have shown that mechanoreceptors are not evenly distributed on the palm as their densities increase towards the fingertips [1,29]. Moreover, the ridges on the palm may have played a role on increasing the intensity of perception for the "large" stimulation shapes. Previous studies indicated that the microstructure of skin strongly affect the perception of skin stretch displays [22].

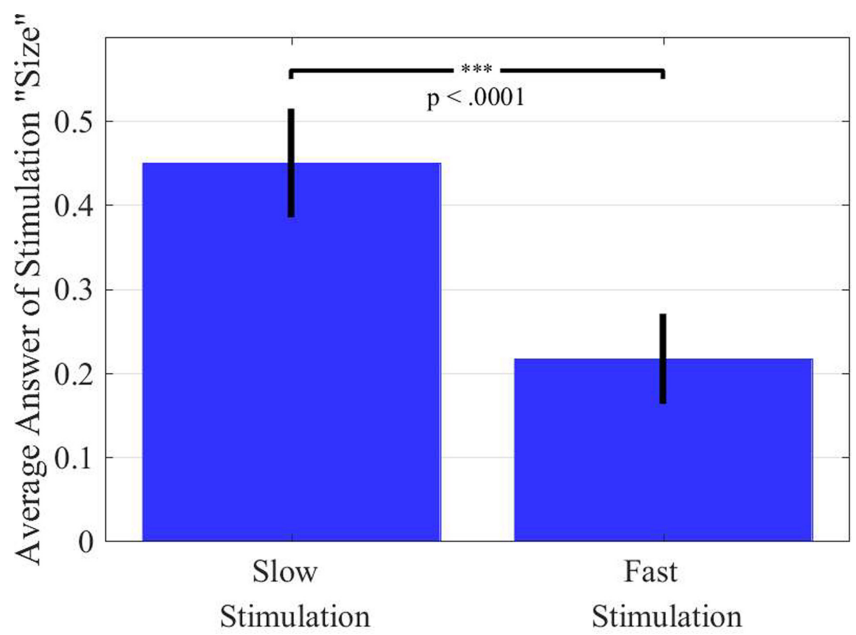

Fig. 7. The means and standard errors of average stimulus sizes at two tactor speeds. The areas created by slow tactor speeds were perceived statistically significantly larger than fast speeds.

Despite the statistical differences between stimuli sizes, the experiments showed that the "circle" stimulation shape had statistically significantly less intensity than the "square" and "triangle" as illustrated in Fig. 6. It seems that the presence of corners in the latter shapes amplified the intensity of stimulation even though the tactor did not stop at those corners during the experiments. These corners might have increased the friction coefficient between the tip of the tactor and skin, thus, increasing the intensity. It has been shown that tangential displacements with friction is perceived with higher sensitivity than frictionless motion [19,28]. Moreover, the triangular shape was perceived with the highest intensity out of the three stimulation shapes. This suggests that acute angles of a stimulus may have an impact on its intensity. Although this effect is distinctly 
different than the priming effect, where multiple stimuli in the same direction increase the sensitivity of perception [11], it is however worth investigating in the future.

In addition to the statistical differences in the intensity between tactor speeds, the stimulation size, and shape, the experimental results showed that "slow" tactor speeds were perceived statistically significantly larger than "fast" tactor speeds even though the 12 experiments were randomly conducted. Whitsel et al. studied the velocity of a stimulus against its perceived motion and found that distances can be perceived shorter as the stimulus velocity increased [31]. Others have concluded that a stimulus length is perceived as result of speed and duration [33]. Such a phenomenon can occur due to the spatial properties of the receptors in the skin [18]. The time duration in our experiments varied from approximately 7 seconds to 15 seconds for each experiment depending on the tactor speed and stimulation shape. It is possible that subjects may have perceived the stimulation shapes of "fast" tactor speeds as smaller. Moreover, seven out of ten subjects perceived the "small" square at "fast" tactor speed as a small "circle". This might be related to the similarities between the small square and circle in the perimeters and areas as shown in Table 1.

\section{Conclusion}

In this paper, the effects of stimulation speed, shape and intensity on skin stretch were investigated by conducting a series of experiments on the palm of the hand. Ten subjects participated in these experiments where two tactor speeds and three stimulation shapes were tested. The results showed that stimuli intensities of relatively slow tactor speeds were statistically significantly less than the fast ones. The experimental results also showed that relatively small stimulation sizes and circular shapes were perceived with less intensity than large stimulation sizes and other shapes respectively. Further, there were also differences in the perceived sizes of stimuli among stimulation shapes. Future work will focus on studying the direction of the stimulus and stimulation angles of different shapes on the skin.

Acknowledgment. The authors would like to acknowledge Al Zaytoonah University of Jordan for funding this research under the grant number 2017-2016/64/17.

\section{References}

1. Asamura, N., Shinohara, T., Tojo, Y., Shinoda, H.: Necessary spatial resolution for realistic tactile feeling display. In: ICRA, pp. 1851-1856. Citeseer (2001)

2. Bark, K., Wheeler, J., Lee, G., Savall, J., Cutkosky, M.: A wearable skin stretch device for haptic feedback. In: World Haptics 2009-Third Joint EuroHaptics Conference and Symposium on Haptic Interfaces for Virtual Environment and Teleoperator Systems, pp. 464-469. IEEE (2009) 
3. Bark, K., Wheeler, J.W., Premakumar, S., Cutkosky, M.R.: Comparison of skin stretch and vibrotactile stimulation for feedback of proprioceptive information. In: 2008 Symposium on Haptic Interfaces for Virtual Environment and Teleoperator Systems, pp. 71-78. IEEE (2008)

4. Biggs, J., Srinivasan, M.A.: Tangential versus normal displacements of skin: relative effectiveness for producing tactile sensations. In: Proceedings 10th Symposium on Haptic Interfaces for Virtual Environment and Teleoperator Systems. HAPTICS 2002, pp. 121-128. IEEE (2002)

5. Caswell, N.A., Yardley, R.T., Montandon, M.N., Provancher, W.R.: Design of a forearm-mounted directional skin stretch device. In: 2012 IEEE Haptics Symposium (HAPTICS), pp. 365-370. IEEE (2012)

6. Chambers, M.R., Andres, K., Duering, M.V., Iggo, A.: The structure and function of the slowly adapting type ii mechanoreceptor in hairy skin. Q. J. Exp. Physiol. Cognate Med. Sci. 57(4), 417-445 (1972)

7. Chen, D.K., Anderson, I.A., Walker, C.G., Besier, T.F.: Lower extremity lateral skin stretch perception for haptic feedback. IEEE Trans. Haptics 9(1), 62-68 (2016)

8. Chinello, F., Pacchierotti, C., Tsagarakis, N.G., Prattichizzo, D.: Design of a wearable skin stretch cutaneous device for the upper limb. In: 2016 IEEE Haptics Symposium (HAPTICS), pp. 14-20. IEEE (2016)

9. Edin, B.B., Essick, G.K., Trulsson, M., Olsson, K.A.: Receptor encoding of moving tactile stimuli in humans. i. temporal pattern of discharge of individual lowthreshold mechanoreceptors. J. Neurosci. 15(1), 830-847 (1995)

10. Gallo, S., Son, C., Lee, H.J., Bleuler, H., Cho, I.J.: A flexible multimodal tactile display for delivering shape and material information. Sensors Actuators A: Phys. 236, 180-189 (2015)

11. Gardner, E.P., Sklar, B.F.: Discrimination of the direction of motion on the human hand: a psychophysical study of stimulation parameters. J. Neurophysiol. 71(6), 2414-2429 (1994)

12. Gleeson, B.T., Horschel, S.K., Provancher, W.R.: Perception of direction for applied tangential skin displacement: effects of speed, displacement, and repetition. IEEE Trans. Haptics 3(3), 177-188 (2010)

13. Guzererler, A., Provancher, W.R., Basdogan, C.: Perception of skin stretch applied to palm: effects of speed and displacement. In: Bello, F., Kajimoto, H., Visell, Y. (eds.) EuroHaptics 2016. LNCS, vol. 9774, pp. 180-189. Springer, Cham (2016). https://doi.org/10.1007/978-3-319-42321-0_17

14. Hayward, V.: Display of haptic shape at different scales. In: Proceedings of Eurohaptics, vol. 2004, pp. 20-27. Citeseer (2004)

15. Johnson, K.O.: The roles and functions of cutaneous mechanoreceptors. Current Opin. Neurobiol. 11(4), 455-461 (2001)

16. Jones, L.A., Ray, K.: Localization and pattern recognition with tactile displays. In: 2008 Symposium on Haptic Interfaces for Virtual Environment and Teleoperator Systems, pp. 33-39. IEEE (2008)

17. Kim, Y., Harders, M., Gassert, R.: Identification of vibrotactile patterns encoding obstacle distance information. IEEE Trans. Haptics 8(3), 298-305 (2015)

18. Nguyen, E.H., Taylor, J.L., Brooks, J., Seizova-Cajic, T.: Velocity of motion across the skin influences perception of tactile location. J. Neurophysiol. 115(2), 674-684 (2015)

19. Olausson, H., Norrsell, U.: Observations on human tactile directional sensibility. J. Physiol. 464(1), 545-559 (1993)

20. Paré, M., Carnahan, H., Smith, A.M.: Magnitude estimation of tangential force applied to the fingerpad. Exp. Brain Res. 142(3), 342-348 (2002) 
21. Petermeijer, S.M., De Winter, J.C., Bengler, K.J.: Vibrotactile displays: a survey with a view on highly automated driving. IEEE Trans. Intell. Transp. Syst. 17(4), 897-907 (2016)

22. Pham, T.Q., Hoshi, T., Tanaka, Y., Sano, A.: Effect of 3D microstructure of dermal papillae on SED concentration at a mechanoreceptor location. PloS One 12(12), e0189293 (2017)

23. Ploch, C.J., Bae, J.H., Ju, W., Cutkosky, M.: Haptic skin stretch on a steering wheel for displaying preview information in autonomous cars. In: 2016 IEEE/RSJ International Conference on Intelligent Robots and Systems (IROS), pp. 60-65. IEEE (2016)

24. Rhodes, J., Clay, C., Phillips, M.: The surface area of the hand and the palm for estimating percentage of total body surface area: results of a meta-analysis. Br. J. Dermatol. 169(1), 76-84 (2013)

25. Schorr, S.B., Okamura, A.M.: Fingertip tactile devices for virtual object manipulation and exploration. In: Proceedings of the 2017 CHI Conference on Human Factors in Computing Systems, pp. 3115-3119. ACM (2017)

26. Shi, S., Leineweber, M.J., Andrysek, J.: Examination of tactor configurations for the design of vibrotactile feedback systems for use in lower-limb prostheses. In: ASME 2018 International Design Engineering Technical Conferences and Computers and Information in Engineering Conference, p. V008T10A006. American Society of Mechanical Engineers (2018)

27. Son, B., Park, J.: Haptic feedback to the palm and fingers for improved tactile perception of large objects. In: The 31st Annual ACM Symposium on User Interface Software and Technology, pp. 757-763. ACM (2018)

28. Sylvester, N.D., Provancher, W.R.: Effects of longitudinal skin stretch on the perception of friction. In: Second Joint EuroHaptics Conference and Symposium on Haptic Interfaces for Virtual Environment and Teleoperator Systems (WHC 2007), pp. 373-378. IEEE (2007)

29. Vallbo, A.B., Johansson, R.S., et al.: Properties of cutaneous mechanoreceptors in the human hand related to touch sensation. Hum. Neurobiol. 3(1), 3-14 (1984)

30. Verrillo, R.T.: Investigation of some parameters of the cutaneous threshold for vibration. J. Acoust. Soc. Am. 34(11), 1768-1773 (1962)

31. Whitsel, B., et al.: Dependence of subjective traverse length on velocity of moving tactile stimuli. Somatosensory Res. 3(3), 185-196 (1986)

32. Yem, V., Shibahara, M., Sato, K., Kajimoto, H.: Expression of 2DOF fingertip traction with 1DOF lateral skin stretch. In: Hasegawa, S., Konyo, M., Kyung, K.U., Nojima, T., Kajimoto, H. (eds.) AsiaHaptics 2016. LNEE, vol. 432, pp. 21-25. Springer, Singapore (2018). https://doi.org/10.1007/978-981-10-4157-0_4

33. Yusoh, S.M.N.S., Nomura, Y., Sakamoto, R., Iwabu, K.: A study on the duration and speed sensibility via finger-pad cutaneous sensations. Procedia Eng. 41, 12681276 (2012)

34. Zhang, M., Turner-Smith, A., Roberts, V.: The reaction of skin and soft tissue to shear forces applied externally to the skin surface. Proc. Inst. Mech. Eng. Part H: J. Eng. Med. 208(4), 217-222 (1994) 
Open Access This chapter is licensed under the terms of the Creative Commons Attribution 4.0 International License (http://creativecommons.org/licenses/by/4.0/), which permits use, sharing, adaptation, distribution and reproduction in any medium or format, as long as you give appropriate credit to the original author(s) and the source, provide a link to the Creative Commons license and indicate if changes were made.

The images or other third party material in this chapter are included in the chapter's Creative Commons license, unless indicated otherwise in a credit line to the material. If material is not included in the chapter's Creative Commons license and your intended use is not permitted by statutory regulation or exceeds the permitted use, you will need to obtain permission directly from the copyright holder. 